\title{
Data-acquisition system developments for ATLAS pixel QA and QC test toward High-Luminosity LHC
}

\author{
Kazuki Todome ${ }^{\mathrm{a}}$, On behalf of the ATLAS ITk collaboration ${ }^{1}$, \\ ${ }^{a}$ INFN Bologna, V.le C. Berti Pichat 6/2 40127 Bologna Italy
}

\begin{abstract}
In preparation for the High-luminosity LHC (HL-LHC) upgrade, the whole ATLAS inner tracker will be replaced by a new silicon detector tracker. The innermost region will be covered by silicon pixel detectors as a high density of produced particles is expected. To operate in such an environment, high-resolution sensors and a high-speed readout system are required. At the moment, the front-end readout chip prototype RD53A and a data-acquisition system (the YARR system) based on a commercial FPGA board and dedicated software for quality assurance (QA) and quality control (QC) test have been developed. Due to the high density of sensor channels, the output speed from RD53A is at maximum 1.28 Gbps per line, sixteen times faster than the readout front-end chip currently used in the ATLAS pixel system. The data-acquisition system needs to establish communication with 1.28 Gbps speed during the QC tests, to validate the data-acquisition path, and to optimize the procedure for data taking at high speed. From the QC perspective this optimization allows to test large numbers of modules simultaneously exploiting the Data AcQuisition (DAQ) speed reducing the time needed for the tests. Another challenging point is the novel concept of readout structure planned for the operation after installation. In HL-LHC, large parts of the ATLAS DAQ system infrastructure are going to be shared among all sub-detectors, using FELIX systems, while current ATLAS DAQ systems are dedicated for each sub-detector. This means that all processes done in the present DAQ system hardware need to be overhauled into software running on the new DAQ system. To minimize the differences between the DAQ system for operation and QC test, we introduced the prototype FELIX system into the DAQ path of the YARR system. In this proceeding, an established DAQ structure for QA and QC test of the new pixel detector is introduced, and results from basic QC tests of the pixel detector with the new readout chip are presented.
\end{abstract}

Keywords: ATLAS, ITk, pixel, FELIX, DAQ

\section{Introduction}

The Large Hadron Collider (LHC) at CERN is the world's highest energy particle accelerator. To collect significantly large amount of data during the next decade after the LHC project, the High Luminosity LHC (HL-LHC) project is planned. At the HL-LHC, the instantaneous luminosity is planned to be increased by a factor of 5 to 7.5 with respect to the LHC design, and accordingly pile-up and radiation damage are expected to be increased significantly. The detectors used for LHC, including ATLAS [1], need to be upgraded to operate in such extreme environments. As part of the

\footnotetext{
${ }^{*}$ Corresponding author. e-mail: kazuki.todome@ cern.ch

Copyright 2020 CERN for the benefit of the ATLAS Collaboration. CC-BY-4.0 license.
}

Preprint submitted to NIMA upgrade of the ATLAS detector, the current inner detector is going to be replaced by new silicon based inner trackers (ITk) consisting of pixel and strip detectors.

While the current pixel system consists of about 2000 modules, the ITk pixel system will be composed of roughly 9200 modules. Those newly designed modules need to pass Quality Assurance (QA) test and Quality Control (QC) test. At the moment, a data-acquisition (DAQ) system, so called YARR (Yet Another Rapid Readout), for QA/QC test of the prototype of front-end readout chip (RD53A) has been developed based on the PCI Express (PCIe) board. Those are introduced in Section 1.1 and Section 1.2.

Although the YARR system is available as a DAQ system for QA/QC test of the new modules, there is a significantly different concept of DAQ system for the operation of the experiment itself. In HL-LHC, large 
parts of the ATLAS DAQ system infrastructure for operation will be shared among all sub-detectors. In particular, the ReadOut Drivers (ROD) will be replaced by the FELIX (Front-End LInk eXchange) system (cf. Section 1.3).

To optimize the DAQ system for operation in early stages, it is ideal to minimize the differences between the DAQ system for operation and QA/QC tests. In this proceeding, a prototype FELIX system is introduced into the DAQ chain of the YARR system in Section 2, and results from basic $\mathrm{QA} / \mathrm{QC}$ tests of the pixel detector are shown in Section 3.

\subsection{RD53A}

RD53A is the prototype of the readout chip [2] produced with $65 \mathrm{~nm}$ technology. The size of the chip is $2 \times 2 \mathrm{~cm}^{2}$, and the readout pixel size is $50 \times 50 \mu \mathrm{m}^{2}$, with a total of $76800(192 \times 400)$ readout pixels. To investigate the best architecture of the analog part of the readout chip, RD53A has three blocks of pixels with different types of front-end circuits. RD53A has three programmable output lanes up to $1.28 \mathrm{Gbps}$, making it 16 times faster than the readout chip currently used in the ATLAS pixel system, with Aurora 64/66 protocol, while the chip is controlled with $160 \mathrm{Mbps}$ single differential serial inputs. Figure 1 shows a single chip card with RD53A.

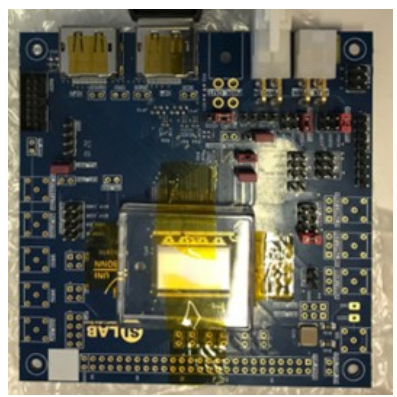

Figure 1: Single chip card with RD53A. The readout chip is located at the bottom in the center of the picture and the display ports for communication are located on top of the picture.

\section{2. $Y A R R$}

YARR is a readout system for several types of readout chips [3]. It can run on commercial FPGA boards such as XpressK7. The key concept of this system is to implement in software all the functionalities that are implemented in firmware in the current DAQ system. The FPGA works simply as an interface to the readout chip. Thanks to this concept, by switching software interface, the system is able to communicate not only with RD53A but also with the readout chip of the current pixel detector (FEI4B) and with the readout chip for ITk Strips (ABC/HCC STAR). An additional advantage is the small latency of the readout chain because of the simple structure of FPGA.

The communication of YARR with RD53A is constructed as follows. The display port from RD53A is connected to the FPGA board via an FPGA Mezzanine Card (FMC) called Ohio card. The FPGA board is mounted on a PC where the software runs, and communicates with the software via PCIe. Basic functionalities for QA/QC tests of RD53A have been developed with the YARR system.

\subsection{FELIX}

FELIX, the infrastructure for the ATLAS DAQ system, will start to be used for readout of some subdetectors already in Run3 (2021) and is expected to be employed by all sub-detectors for HL-LHC operation [4]. In the current ATLAS DAQ system, all sub-detectors have a dedicated DAQ chain, which processes data with protocols that are specific to each subdetector. Those dedicated DAQ chains process data with different protocols depending on the specific subdetectors. It is relatively simple to build this type of DAQ chains, but every dedicated DAQ chain requires individual maintenance. In contrast to the current ATLAS DAQ system, the key concept of FELIX is to share the same readout electronic system between all subdetectors.

For Run 3, FELIX is implemented on FLX712 [4] which is an ATLAS custom board based on a Xilinx Kintex UltraScale FPGA (Figure 2). It has 48 optical links to communicate with detectors, and 16 PCIe lanes to communicate with the host PC. Uplink of this system lies a 4.6 Gbps GigaBit Transceiver (GBT) protocol [5], and downlink there is either a GBT mode protocol or a custom protocol for Felix, called full-mode protocol.

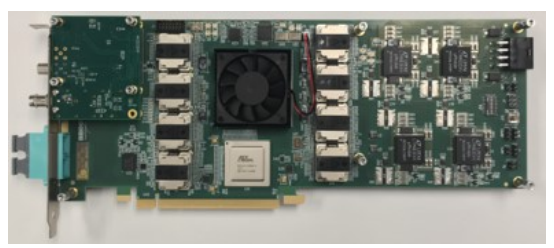

Figure 2: Picuture of a FLX712 board.

For development of the readout chain, a scaled down system is also available. It is implemented on VC709 
which is a Xilinx evaluation board (Figure 3). It has 4 optical links to communicate with detectors, and 8 PCIe lanes to communicate with the host PC. The functionalities of the two systems are the same except for the number of communication lines. The readout chain described here has been constructed with VC709.

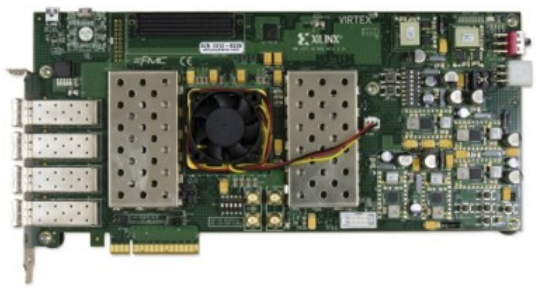

Figure 3: Picuture of a VC709 board.

\section{Readout chain}

As mentioned in Section 1, it is ideal to minimize the differences between the DAQ systems for operation and QA/QC test. Therefore, FELIX boards are introduced in the YARR system as an additional interface. Since input and output protocols are different between RD53A and FELIX, those cannot communicate directly. In this section, two complementary approaches to establish communication are introduced.

One approach is to use a board called $\pi$ LUP (PIxel detector high Luminosity Upgrade card) [6], which is shown in Figure 4. This board is equipped with a Xilinx Kintex-7 and Zynq-7 FPGA. It is connected to RD53A via a Low Pin Count (LPC) FMC connector using the Ohio card, as used in the YARR setup. Communication to FELIX is established via Small Formfactor Pluggable (SFP) connectors by optical fiber. The $\pi$ LUP board works as protocol converter between FELIX and RD53A. The commands generated by software are transmitted to FELIX and are converted to optical signals with a GBT protocol. Received commands on $\pi$ LUP are decoded from the GBT protocol and reconverted into a differential serial signal. The reconverted command is sent to RD53A via a display port. For downlink, the output from RD53A is configured to 160 Mbps with 4 lines in this setup. The output is transmitted with the Aurora protocol from RD53A to $\pi \mathrm{LUP}$, and is decoded on $\pi$ LUP. The decoded output is converted to full-mode protocol and transmitted via optical fiber to the FELIX system.

This setup allows to use the FELIX system without any necessary modification in the firmware, since all

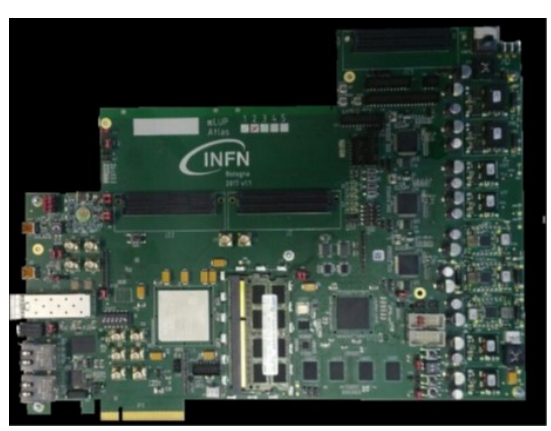

Figure 4: $\pi$ LUP card.

necessary functionalities for protocol conversion are on $\pi$ LUP. The higher speed mode of downlink from $\pi$ LUP (full-mode) is supported. However, for downlink from RD53A, the speed is determined by $160 \mathrm{Mbps}$ in 4 lines, which is smaller than the maximum possible speed.

The other approach is to use a Versatile Link Demo Board (VLDB) [7] and Interface Card (IC), shown in Figure 5 and Figure 6 respectively. The communication structure is shown in Figure 7. Commands from FELIX are transmitted via optical fibers, which are distributed to ICs via HDMI, and then are propagated to RD53A via a display port. Downlink, the output from RD53A is configured to $1.28 \mathrm{Gbps}$ with 1 line in this setup. The outputs are converted from electrical to optical signals without logical conversion on ICs, and are propagated to FELIX. All necessary logical conversion done on $\pi$ LUP is performed in the FELIX firmware interface.

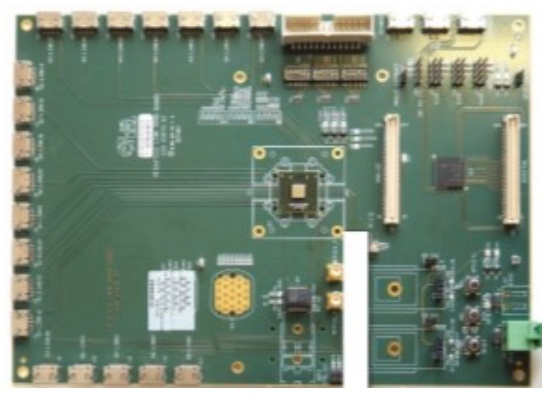

Figure 5: VLDB board.

This setup allows to test several RD53As simultaneously with the highest downlink speed from RD53A. However, downlink of FELIX, the GBT mode is used, with a lower speed. In addition, the FELIX firmware needs to be modified to implement logical conversion.

In both setups, the only modified part in the YARR software is the interface with FELIX. Therefore, all 


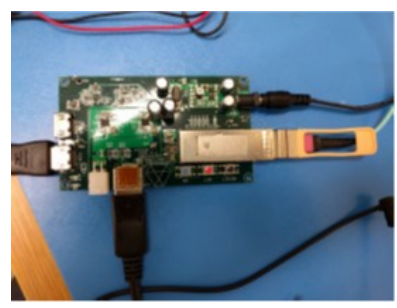

Figure 6: Interface Card (IC).

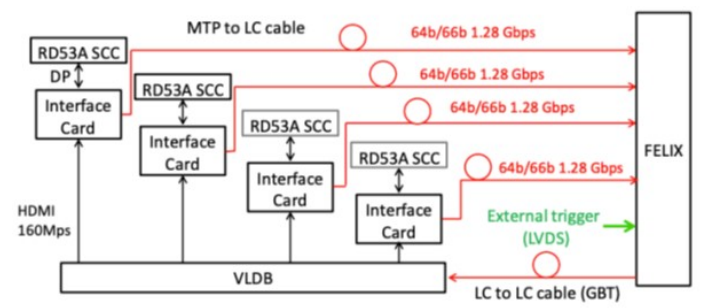

Figure 7: VLDB + ICs communication structure.

functionalities of YARR developed for QA/QC tests of RD53A are available with the setups. In the following section, preliminary results obtained with those two complementary approaches are shown.

\section{Results}

The communication with RD53A is successful with both setups. With a digital scan, which tests the digital circuits using 100 test pulses, $100 \%$ response is received, therefore stable communication is confirmed.

In a threshold scan, a variable charge configured by a register named Vcal is injected to each pixel configured with fixed thresholds. The number of times the single pixel is above threshold as a function of the injected charge is shown in the s-curve (Figure 8). In following tests, only one of the three front-end circuits, the differential front-end which is the selected type of front-end circuits for the ATLAS pixel detector in HLLHC, is activated. From the s-curve, a threshold value is defined from the injection charge with which a $50 \%$ response rate is obtained, and it is converted to electron charges for each pixel. Figure 9 shows the distribution of threshold before the threshold scan: it has typically a wide range before tuning.

The threshold configuration is tuned so that all pixels have the same threshold. The threshold tuning is performed by using the occupancy distribution, which is shown in Figure 10, with various threshold configu-

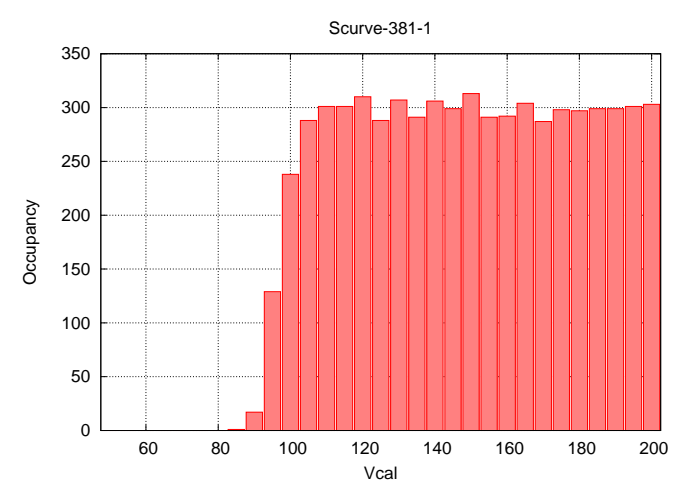

Figure 8: An example of s-curve for a pixel. A number of hit counts with various injection charges controlled by the register named Vcal is shown. In this case, The threshold value is defined from Vcal $=96$.

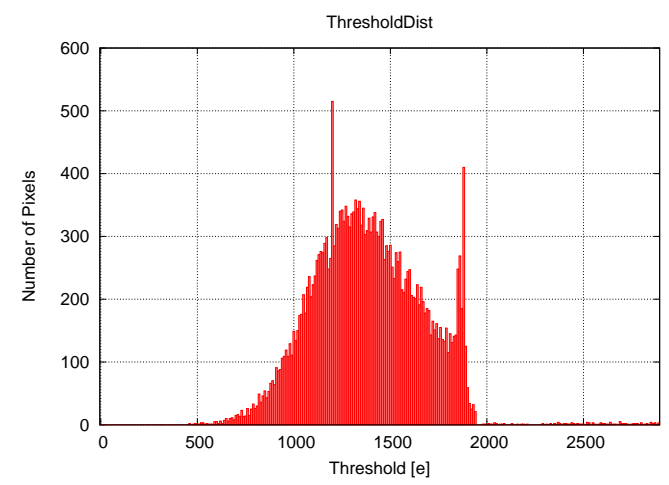

Figure 9: Threshold distribution before threshold tuning. It is widely distributed from around 500 to 2000 electron charges.

rations and with an injection charge fixed to the target value. The threshold configuration is tuned to have an occupancy distribution of $50 \%$ for the fixed target injection charge. Since the threshold value is defined by the injection charge with $50 \%$ of occupancy, after a successful threshold tune, the threshold distribution converges around the target value. Figure 11 shows an example of a threshold distribution after threshold tuning with 1000 electron charges as the target value. Although few pixels have a larger threshold because of failure in fitting the s-curve, most pixels have a threshold close to the target value.

\section{Conclusion}

Two setups for QA/QC test of RD53A with FELIX are introduced in this proceeding: one is without modification and in full-mode, the other one is able to be 


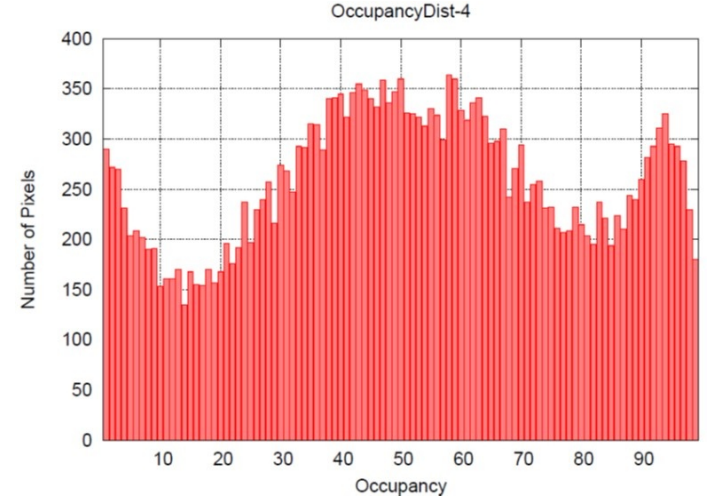

Figure 10: Occupancy distribution during threshold tuning taken with the target injection charge. After succeeded tuning, the occupancy distribution converges around $50 \%$.

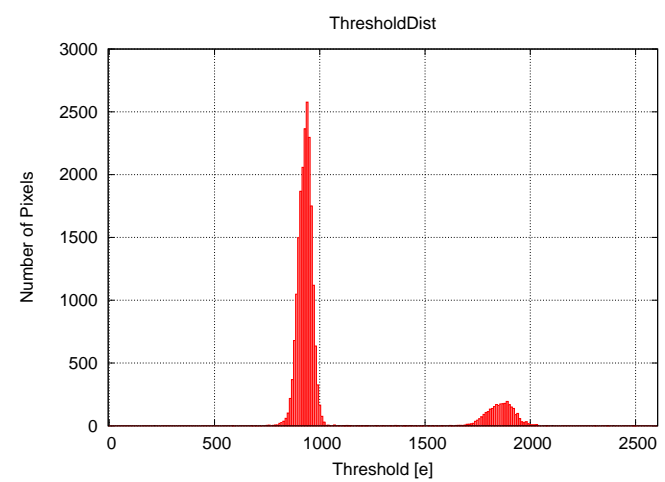

Figure 11: Threshold distribution after threshold tuning. Most of pixels are distributed around the target value of $1000 \mathrm{e}$, while a small amount of pixels can be found around 1900 e because of failure in fitting the s-curve.

used with several RD53As with a maximum speed from RD53A. Digital scan, threshold scan, and threshold tuning are demonstrated with both setups. Both show stable communication and good performance with FELIX.

\section{References}

[1] The ATLAS Collaboration, The ATLAS Experiment at the CERN Large Hadron Collider, JINST 3 (2008) S08003.

[2] M. Garcia-Sciveres, The RD53A Integrated Circuit (CERNRD53-PUB-17-001) (2017).

URL https : //cds . cern. ch/record/2287593

[3] T. Heim, YARR - a PCIe based readout concept for current and future ATLAS pixel modules, J.Phys,: Conf.Ser. 898 (2017) 032053.

[4] J. Anderson, et al., FELIX: a PCIe based high-throughput approach for interfacing front-end and trigger electronics in the ATLAS upgrade framework, JINST 11 (2016) C12023.
[5] P. Moreira, et al., The GBT: A proposed architecure for multi$\mathrm{Gb} / \mathrm{s}$ data transmission in high energy physics (2007).

URL https : //cds . cern. ch/record/1091474

[6] N. Giangiacomi, et al., General Purpose Readout Board $\pi$-LUP: Overview and Results., IEEE Trans. Nucl. Sci. 66 (7) (2019) 1021-1027. arXiv:1806.08858.

[7] R. Martín Lesma and others, The versatile link demo board (VLDB), JINST 12 (2017) C02020. 